\title{
Defining the Incremental Utility of Prostate Multiparametric Magnetic Resonance Imaging at Standard and Specialized Read in Predicting Extracapsular Extension of Prostate Cancer
}

\author{
Kae Jack Tay ${ }^{a,}$, Rajan T. Gupta ${ }^{b}$, Alison F. Brown ${ }^{b}$, Rachel K. Silverman ${ }^{c}$, Thomas J. Polascik $^{a}$

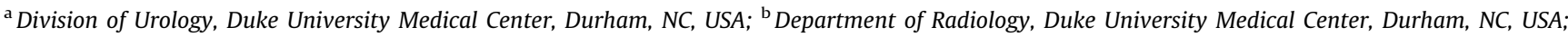 \\ ${ }^{\mathrm{c}}$ Department of Biostatistics, The University of North Carolina at Chapel Hill, Chapel Hill, NC, USA
}

\section{Article info}

Article history:

Accepted October 20, 2015

Associate Editor:

Matthew Cooperberg

Keywords:

Active surveillance

Diagnostic accuracy

Extracapsular extension

Focal therapy

Likelihood

mpMRI

Nerve sparing

Prostate cancer

Sensitivity

Specificity

Utility

\begin{abstract}
Multiparametric magnetic resonance imaging (mpMRI) is increasingly used in staging early prostate cancer (PCa) but remains heavily reader-dependent. We aim to define the incremental utility of mpMRI over clinical parameters in determining the pathologic extracapsular extension (pECE) of PCa interpreted in a standard radiologic setting and when further over-read by a specialized reader. We retrospectively reviewed 120 men with clinically localized PCa undergoing mpMRI and radical prostatectomy. We obtained radiologic prediction of pECE from standard radiologic reports (standard read) and by a specialized reader blinded to clinical and pathologic findings (specialized read). We determined the incremental benefit of standard read and specialized read by sequential addition to a baseline clinical parameters-only logistic regression model predicting pECE. The sensitivity and specificity of standard read were $77 \%$ and $44 \%$, respectively, whereas those of specialized read were $86 \%$ and $81 \%$. The positive likelihood ratio was 1.7 at baseline, 1.7 adding standard read, and 6.5 adding specialized read. The negative likelihood ratio was 0.6 at baseline, 0.5 adding standard read, and 0.1 adding specialized read. Standard read modestly improved prediction of pECE, whereas specialized read improved it moderately.

Patient summary: The incremental benefit of mpMRI over clinical information is small but increases to moderate with a specialized second opinion. This second opinion may be useful when considering active surveillance, nerve-sparing surgery, or focal therapy. (C) 2015 European Association of Urology. Published by Elsevier B.V. All rights reserved.
\end{abstract}

\footnotetext{
* Corresponding author. Division of Urology, Department of Surgery, Duke University Medical Center, Box 2804, Durham, NC 27710, USA. Tel. +1 9196845204.

E-mail address: kae.jack.tay@duke.edu (K.J. Tay).
}

In parallel with a worldwide increase in prostate cancer (PCa) incidence, there has been a surge in patient expectation to preserve potency and continence while preventing morbidity and mortality [1,2]. On the one hand, a spectrum of glandsparing modalities has arisen to meet this need, particularly in men with low-risk or low-volume intermediate-risk cancer. On the other hand, traditional whole-gland treatments are reserved for appropriate candidates with intermediate- and high-risk cancers. Ultimately, the optimal management of PCa depends on accurate risk stratification to avoid delay in intervention for those who need it and to defer treatment in those who can be observed safely. 
Table 1 - Comparison of models with incremental multiparametric magnetic resonance imaging information

\begin{tabular}{|c|c|c|c|c|c|c|}
\hline & Sensitivity, \% & Specificity, \% & Accuracy, \% & $\mathrm{LR}+$ & LR- & AUC ${ }^{*}$ \\
\hline Clinical & 60.0 & 63.6 & 61.7 & 1.65 & 0.63 & $0.69(0.53-0.84)$ \\
\hline Clinical plus MRI standard read ${ }^{\dagger}$ & 68.0 & 59.1 & 63.8 & 1.66 & 0.54 & $0.72(0.57-0.87)$ \\
\hline Clinical plus MRI standard read plus MRI specialized read ${ }^{\ddagger}$ & 88.0 & 86.4 & 87.2 & 6.45 & 0.14 & $0.91(0.82-0.99)$ \\
\hline $\begin{array}{l}\text { AUC = area under the curve; LR+= positive likelihood ratio; } \\
\text { Parentheses indicate } 95 \% \text { confidence intervals. } \\
\text { Not statistically significant compared to prior model. } \\
\ddagger \text { Statistically significant compared to prior model. }\end{array}$ & negative like & d ratio; MRI = & hetic reson & nagi & & \\
\hline
\end{tabular}

Multiparametric magnetic resonance imaging (mpMRI) can more accurately stage PCa compared with traditional parameters [3,4]. Although now widely accepted and available for PCa staging, mpMRI is expensive and significantly affects health care costs $[5,6]$. The diagnostic performance of mpMRI is affected by technical considerations and heavily depends on reader experience [7]. In situations of uncertainty, a tertiary referral sought for a specialized review adds further cost to PCa management.

We sought to define the incremental utility of mpMRI over clinical parameters in determining ECE when read in a standard academic radiologic setting and when further over-read by a dedicated reader with a special interest in prostate mpMRI.

Our detailed methods are reported in Supplement 1. In brief, we reviewed all men undergoing radical prostatectomy for clinically localized PCa and preoperative mpMRI at our tertiary academic institution. The reference standard for ECE was defined by pathologic examination of the prostatectomy specimen ( $\mathrm{pECE}$ ). Radiologic prediction of pECE was obtained from standard radiologic reports (standard read) and by a dedicated reader with special interest in prostate mpMRI blinded to clinical/pathologic and standard-read findings (specialized read). We determined the incremental benefit of standard read and specialized read in predicting pECE by sequentially adding them to a baseline clinical parameters-only logistic regression model trained (using bootstrapping) on a derivation data set and applied to an independent validation data set. All analyses used a significance level of 0.05 and were generated in SAS version 9.3 (SAS Institute, Cary, NC, USA) and $\mathrm{R}$ version 3.1.1 ( $\mathrm{R}$ Foundation for Statistical Computing, Vienna, Austria).

The total cohort included 120 men, of whom 56 (46.7\%) had pECE. Their demographic details are summarized in Supplementary Table 1 . At univariate analysis, the accuracy (fraction of correct ECE classifications) of the mpMRI standard read was $58.7 \%$ (area under the receiver operating characteristic curve $[A U C]=0.60)$, and the accuracy of the specialized read was $82.6 \%($ AUC $=0.83)$ (Supplementary Table 2).

The baseline clinical parameters-only logistic regression model is detailed in Supplementary Table 3. The performance characteristics of the baseline clinical parametersonly model and that of the models sequentially adding mpMRI standard read, and then mpMRI specialized read are shown in Table 1 and Figure 1. In general, a diagnostic test with a likelihood ratio $>10$ or $<0.1$ has a high impact on post-test probability, whereas a test with a likelihood ratio of 5-10 or $0.1-0.2$ is considered to have a moderate impact [8]. The addition of the mpMRI standard read did not significantly improve classification of pECE compared with the first, clinical parameters-only model, culminating in a small observed improvement in the AUC (0.69 to 0.72) and in the negative likelihood ratio ( 0.6 to 0.5$)$. In contrast, the third model, which had sequential addition of the mpMRI specialized read, significantly improved the classification of pECE over the second model $(p<0.001)$. The further specialized read led to a clinically significant improvement in the AUC from 0.72 to 0.91 , in the positive likelihood ratio from 1.7 to 6.5 , and in the negative likelihood ratio from 0.5 to 0.1 . It thus appears that the observed impact of mpMRI at standard reading was small, whereas that of a specialized read was at least moderately significant in influencing the post-test probability of pECE.

Contemporary mpMRI series have reported accuracy of $62-74 \%$, sensitivity of $35-63 \%$, and specificity of $73-91 \%$ in the detection of ECE across a range of inclusion criteria (Supplementary Table 4). In our series, the accuracy of

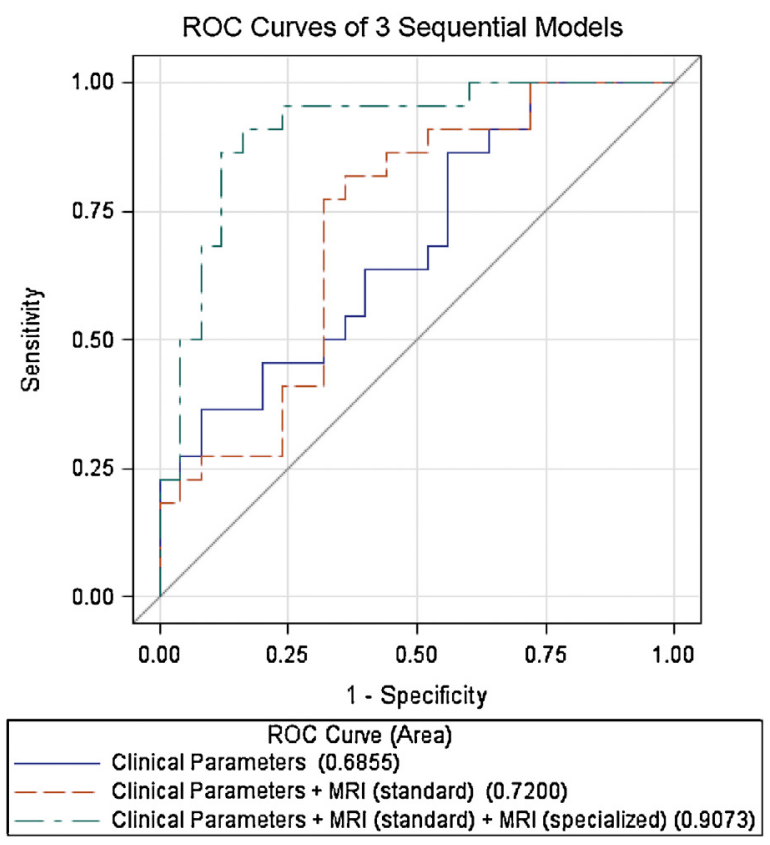

Fig. 1 - Receiver operating characteristic (ROC) curves of all three models. ROC curves for the clinical parameters model and each of the subsequent models adding magnetic resonance imaging at standard read and then specialized read.

$M R I$ = magnetic resonance imaging; $R O C=$ receiver operating characteristic. 
mpMRI at standard read as a stand-alone test was at the lower end of the spectrum, whereas the specialized read performed exceedingly well. At closer examination, it appears that the main difference lies overwhelmingly in the specificity of the tests ( $44 \%$ vs $81 \%$ ), whereas their sensitivities were comparable ( $77 \%$ vs $86 \%$ ). The improved discrimination thus occurs in men who test positive with mpMRI: Those reading "positive" on standard read have a higher probability of not harboring ECE, whereas this likelihood is reduced with specialized read. Specialized read may thus be valuable to men who are potent and keen for a nerve-sparing treatment, whether radical, focal, or expectant. Conversely, those who have already decided for wide resection of periprostatic tissues based on other considerations may benefit less from a specialized read.

The incremental benefit of a specialized read is congruent with findings reported in contemporary literature. MRI interpretation is highly dependent on reader experience, with a Cohen's Kappa as low as 0.0129 between an experienced and inexperienced reader [7,9]. In another learning curve study, Latchamsetty et al reported an improvement in sensitivity from $31 \%$ to $65 \%$ and in specificity from $65 \%$ to $71 \%$ in the detection of ECE between their first 40 and later 40 MRIs read [10]. These data support the notion of specific training and standardized reporting guidelines for radiologic interpretation of ECE. As interest in nerve-sparing and gland-sparing techniques grows, a close relationship between the urologist and radiologist and dedicated training in prostate mpMRI interpretation for both partners become increasingly important.

The strengths of this study are the availability of a pathologic reference as the gold standard and the blinding of our single specialized reader to clinical/pathologic information. Limitations include selection bias in this cohort of men all undergoing prostatectomy, the assumption that our baseline clinical model truly simulates prempMRI physician-predictive ability, and reproducibility given the single-reader nature of the specialized read. A detailed discussion is offered in Supplement 2.

Clinical information remains important in predicting ECE. There is a small benefit with mpMRI, and this benefit becomes moderately important with a specialized read. A specialized read may be pertinent when considering nervesparing surgery or other gland-sparing approaches.

Author contributions: Kae Jack Tay had full access to all the data in the study and takes responsibility for the integrity of the data and the accuracy of the data analysis.

Study concept and design: Tay, Gupta, Polascik.

Acquisition of data: Brown, Gupta, Tay.

Analysis and interpretation of data: Tay, Gupta, Silverman.

Drafting of the manuscript: Tay.

Critical revision of the manuscript for important intellectual content: Gupta, Polascik, Silverman, Brown.

Statistical analysis: Tay, Silverman.

Obtaining funding: None.

Administrative, technical, or material support: None.

Supervision: Polsacik, Gupta.

Other (specify): None.
Financial disclosures: Kae Jack Tay certifies that all conflicts of interest, including specific financial interests and relationships and affiliations relevant to the subject matter or materials discussed in the manuscript (eg, employment/affiliation, grants or funding, consultancies, honoraria, stock ownership or options, expert testimony, royalties, or patents filed, received, or pending), are the following: Dr Kae Jack Tay received fellowship funding from the National Medical Research Council, Singapore. Dr Rajan Gupta has consulted for Invivo. Dr Thomas Polascik has consulted for Endocare and received grant funding from Genomic Health.

Funding/Support and role of the sponsor: None.

\section{Appendix A. Supplementary data}

Supplementary data associated with this article can be found, in the online version, at http://dx.doi.org/10.1016/j. eururo.2015.10.041.

\section{References}

[1] Diefenbach MA, Mohamed NE. Regret of treatment decision and its association with disease-specific quality of life following prostate cancer treatment. Cancer Invest 2007;25:449-57.

[2] Schroeck FR, Krupski TL, Stewart SB, et al. Pretreatment expectations of patients undergoing robotic assisted laparoscopic or open retropubic radical prostatectomy. J Urol 2012;187:894-8.

[3] Gupta RT, Faridi KF, Singh AA, et al. Comparing 3-T multiparametric MRI and the Partin tables to predict organ-confined prostate cancer after radical prostatectomy. Urol Oncol 2014;32:1292-9.

[4] Woo S, Cho JY, Kim SY, Kim SH. Extracapsular extension in prostate cancer: added value of diffusion-weighted MRI in patients with equivocal findings on T2-weighted imaging. AJR Am J Roentgenol 2015;204:W168-75.

[5] Lotan Y, Haddad AQ Costa DN, Pedrosa I, Rofsky NM, Roehrborn CG. Decision analysis model comparing cost of multiparametric magnetic resonance imaging vs. repeat biopsy for detection of prostate cancer in men with prior negative findings on biopsy. Urol Oncol 2015;33, 266.e9-16.

[6] de Rooij M, Crienen S, Witjes JA, Barentsz JO, Rovers MM, Grutters JP. Cost-effectiveness of magnetic resonance (MR) imaging and MR-guided targeted biopsy versus systematic transrectal ultrasound-guided biopsy in diagnosing prostate cancer: a modelling study from a health care perspective. Eur Urol 2014;66: 430-6.

[7] Ruprecht O, Weisser P, Bodelle B, Ackermann H, Vogl TJ. MRI of the prostate: interobserver agreement compared with histopathologic outcome after radical prostatectomy. Eur J Radiol 2012;81: 456-60.

[8] Jaeschke R, Guyatt GH, Sackett DL. Users' guides to the medical literature. III. How to use an article about a diagnostic test. B. What are the results and will they help me in caring for my patients? The Evidence-Based Medicine Working Group. JAMA 1994;271: 703-7.

[9] Otto J, Thormer G, Seiwerts M, et al. Value of endorectal magnetic resonance imaging at $3 \mathrm{~T}$ for the local staging of prostate cancer. Rofo 2014;186:795-802.

[10] Latchamsetty KC, Borden Jr LS, Porter CR, et al. Experience improves staging accuracy of endorectal magnetic resonance imaging in prostate cancer: what is the learning curve? Can J Urol 2007;14: 3429-34. 